\title{
Heavy Flavor Production in Heavy lon Collisions at CMS
}

\author{
Jian Sun on behalf of the CMS Collaboration*t \\ Purdue University (US) \\ E-mail: sun229@purdue.edu
}

Studies of Heavy flavor production are of great interest in heavy ion collisions. In the produced medium, the binding potential between a quark and antiquark in quarkonium is screened by surrounding light quarks and antiquarks. Thus, the various quarkonium states are expected to be melt at different temperatures depending on their binding energies, which allows us to characterize the QCD phase transition. In addition, open heavy flavor production are relevant for flavor-dependence of the in-medium parton energy loss. In QCD, gluons are expected to lose more energy compared to quarks when passing through the QGP due to the larger color charge. Compared to light quarks, heavy quarks are expected to lose less radiative energy because gluon radiation is suppressed at angles smaller than the ratio of the quark mass to its energy. This "dead cone effect" (and its disappearance at high transverse momentum) can be studied using open heavy flavor mesons and heavy flavor tagged jets. With CMS detector, quarkonia, open heavy flavor meson and heavy flavor tagged jet spectra are studied with high precision. In this talk, recent results from $\mathrm{pp}, \mathrm{pPb}$ and $\mathrm{PbPb}$ at 2.76 and $5.02 \mathrm{TeV}$ collisions are presented.

38th International Conference on High Energy Physics

3-10 August 2016

Chicago, USA

* Speaker.

${ }^{\dagger}$ A list of members of the CMS Collaboration and acknowledgements can be found at the end of this issue. 


\section{Introduction}

Because of their large mass, heavy quarks are primarily produced at early stages of heavyion collisions, and therefore experience the full evolution of the system, which makes them good probes of the Quark-Gluon plasma (QGP). The productions of various quarkonium states are expeced to be suppressed by the screening effect [1]. Furthermore, the various quarkonium states are expected to be melt at different temperatures depending on their binding energies, which allows us to characterize the QCD phase transition. In addition, open heavy flavor production are relevant for flavor-dependence of the in-medium parton energy loss. Compared with light quarks and gluons, heavy quarks are expected to lose less energy due to color charge and dead cone effect [2]. Furthermore, study of heavy quarks can also help us understand the cold nuclear matter effects [3].

The CMS experiment is a general-purpose detector with large acceptance and good performance on reconstruciton of track, muon, jets, etc. In CMS, heavy quarkonium is mainly studied through dilepton decay channels. The open heavy flavor is studied through reconstructed $D$ and $B$ hadrons, $J / \psi$ from $B$ hadron decay, and heavy flavor jets. In this article, Sec. 2 and 3 present the measurements of quarkonia and open heavy flavor with CMS, respectively.

\section{Quarkonia Measurements}

The left panel of Figure 1 shows the $\Upsilon(n S) / \Upsilon(1 S)$ ratio as a function of event multiplicity in 2.76 TeV pp and $5.02 \mathrm{TeV}$ pPb collisions [4]. The $\Upsilon(n S) / \Upsilon(1 S)$ ratio is found to decrease toward higher multiplicity, which indicates there are effects in small systems to be further studied. The right panel shows the prompt $J / \psi R_{F B}=\frac{d^{2} \sigma\left(\mathrm{y}_{\mathrm{CM}}>0\right) / \mathrm{dp}_{\mathrm{T}} \mathrm{dy}_{\mathrm{CM}}}{d^{2} \sigma\left(\mathrm{y}_{\mathrm{CM}}<0\right) / \mathrm{dp}_{\mathrm{T}} \mathrm{dy}_{\mathrm{CM}}}$ as a function of $p_{T}$ in $\mathrm{pPb}$ collisions at $5.02 \mathrm{TeV}$ [5]. The prompt $J / \psi$ production in $\mathrm{y}_{\mathrm{CM}}>0$ region is found to be suppressed compared with $\mathrm{y}_{\mathrm{CM}}<0$ region, which is due to cold nuclear matter effects or a combination of cold nuclear matter effects and other effects.
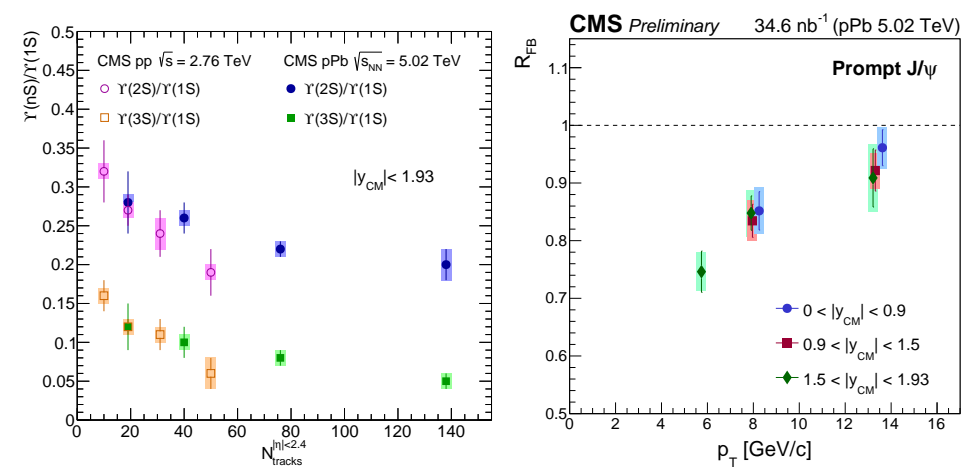

Figure 1: (Left) $\Upsilon(n S) / \Upsilon(1 S)$ ratio as a function of $N_{\text {tracks }}^{|\eta|<2.4}$ in $2.76 \mathrm{TeV}$ pp and $5.02 \mathrm{TeV} \mathrm{pPb}$ collisions for $\Upsilon(2 S)$ and $\Upsilon(3 S)$ in $\left|\mathrm{y}_{\mathrm{CM}}\right|<1.93$. (Right) Prompt $J / \psi R_{F B}$ as a function of $p_{T}$ in different $\mathrm{y}_{\mathrm{CM}}$ regions.

Figure 2 shows prompt $J / \psi R_{A A}$ (left) and $v_{2}$ (right) as a function of $N_{\text {part }}$ for the $p_{T}$ range 6.5 GeV/c to $30.0 \mathrm{GeV} / \mathrm{c}$ in $\mathrm{PbPb}$ collisions at $2.76 \mathrm{TeV}$ [6]. The prompt $J / \psi$ production is significantly suppressed in $\mathrm{PbPb}$ collisions and the suppression has a clear centrality dependence. The prompt $J / \psi v_{2}$ is found to be positive in studied $p_{T}$ range, which indicates the path length dependence of energy loss. 

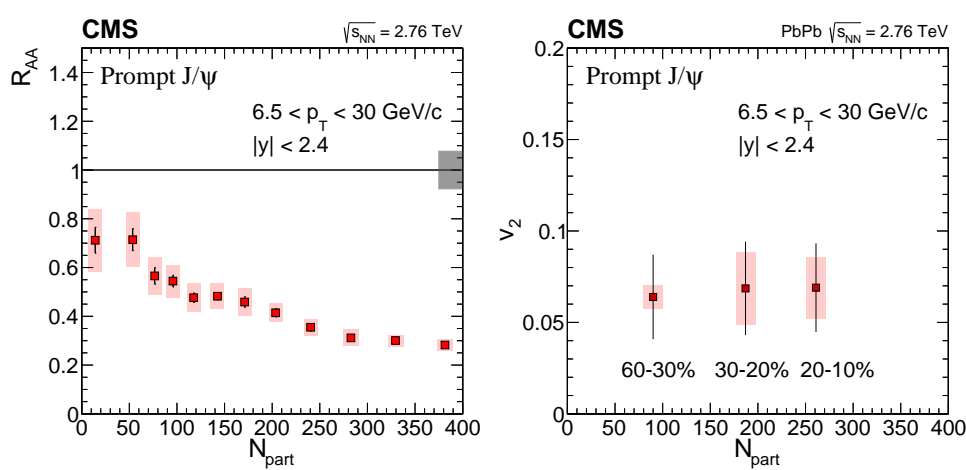

Figure 2: Prompt $J / \psi R_{A A}$ (left) and $v_{2}$ (right) as a function of $N_{\text {part }}$ in $\mathrm{PbPb}$ collisions at $2.76 \mathrm{TeV}$.

Figure 3 shows $R_{A A}$ of $\Upsilon(1 S)$ and $\Upsilon(2 S)$ as functions of $p_{T}$ (left) and $N_{\text {part }}$ (right) in $\mathrm{PbPb}$ collisions at $2.76 \mathrm{TeV}$ [7]. The $R_{A A}$ of $\Upsilon(2 S)$ is smaller than the $R_{A A}$ of $\Upsilon(1 S)$, which is consistent with the fact that the binding energy of $\Upsilon(1 S)$ is bigger than that of $\Upsilon(2 S)$. The suppression of $\Upsilon(1 S)$ and $\Upsilon(2 S)$ is found to have a clear centrality dependence and little dependence on $p_{T}$.
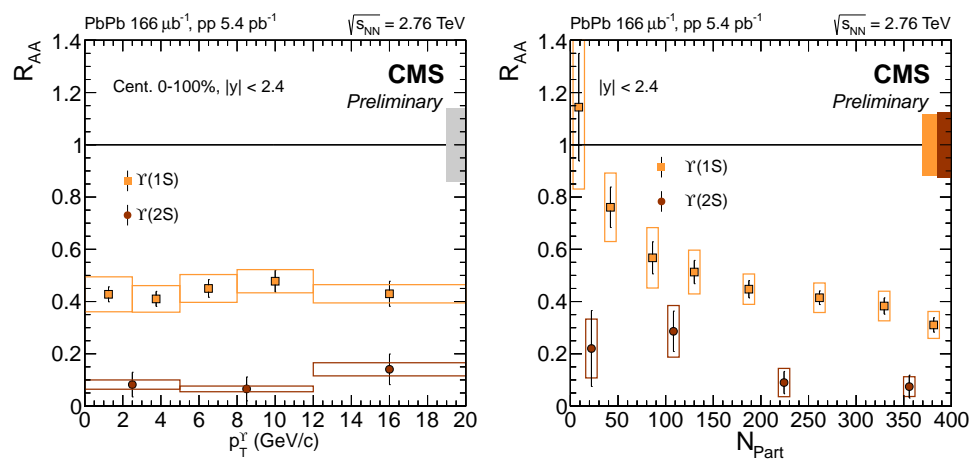

Figure 3: $R_{A A}$ of $\Upsilon(1 S)$ and $\Upsilon(2 S)$ as functions of $p_{T}$ (left) and $N_{\text {part }}$ (right) in $\mathrm{PbPb}$ collisions at 2.76 TeV.

\section{Open Heavy Flavor Measurements}
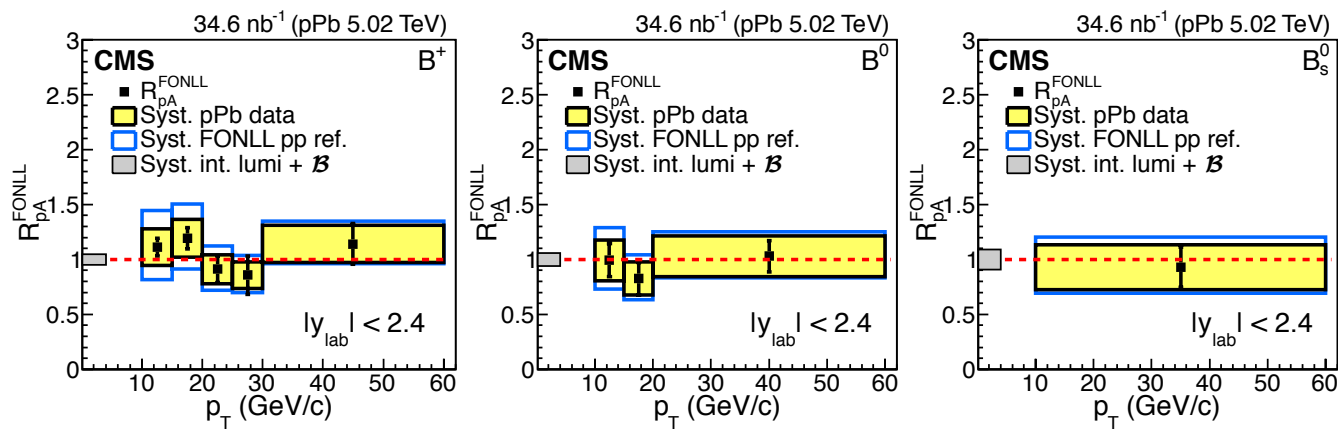

Figure 4: $R_{p A}^{F O N L L}$ of $B^{+}$(left), $B^{0}$ (middle) and $B_{s}^{0}$ (right) mesons in $\left|\mathrm{y}_{\text {lab }}\right|<2.4$ as a function of $p_{T}$ in $\mathrm{pPb}$ collisions at $5.02 \mathrm{TeV}$. 

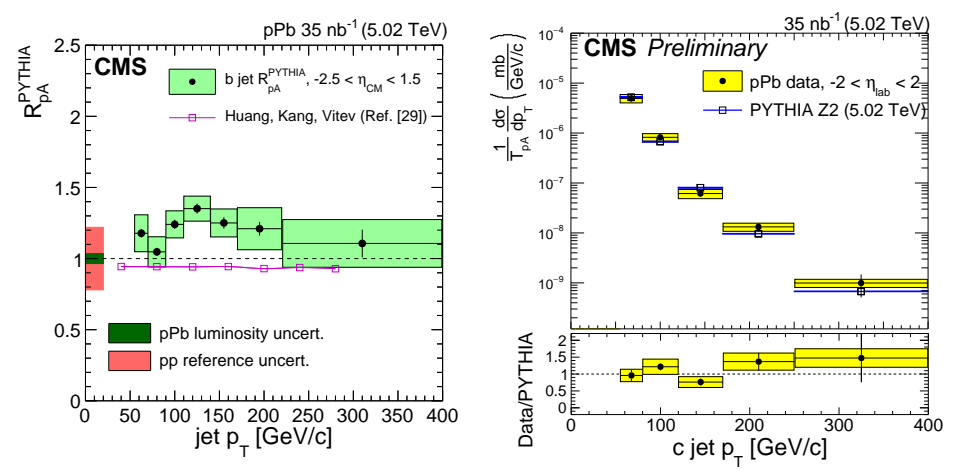

Figure 5: (Left) b-jet $R_{p A}^{P Y T H I A}$ as a function of $p_{T}$ in $\mathrm{pPb}$ collisions at $5.02 \mathrm{TeV}$. (Right) c-jet invariant yield scaled by $T_{p A}$ as a function of $p_{T}$ in $\mathrm{pPb}$ collisions at $5.02 \mathrm{TeV}$. PYTHIA calculation is also shown for comparison.

The cold nuclear matter effects are studied with the B mesons, b-jet and c-jet production in $\mathrm{pPb}$ collisions at $5.02 \mathrm{TeV}$. The $R_{p A}^{F O N L L}$ of B mesons is found to be consistent with unity within uncertainties as showed in Figure 4 [8]. The $R_{p A}^{P Y T H I A}$ of b-jet and c-jet is also found to be consistent with unity within uncertainties as showed in Figure 5 [9]. Thus, no significant cold nuclear matter effects are observed in these measurements and cold nuclear matter effects should not play an important role in heavy flavor production at high $p_{T}$ in AA collisions.

Figure 6 shows prompt $D^{0} R_{A A}$ as a function of $p_{T}$ for centrality classes $0-100 \%$ (left) and $0-10 \%$ (right) in $\mathrm{PbPb}$ collision at $5.02 \mathrm{TeV}$ [10]. Compared with previous measurement of prompt $D^{0} R_{A A}$ at $2.76 \mathrm{TeV}$ with CMS [11], the significant improvement is that the reference of the new $R_{A A}$ results is measured pp reference at the same energy. The suppression of $D^{0}$ at $5.02 \mathrm{TeV}$ is found to be consistent with the suppression at $2.76 \mathrm{TeV}$. It is found that the prompt $D^{0} R_{A A}$ is close to charged particle $R_{A A}$ and they have similar $p_{T}$ dependence.
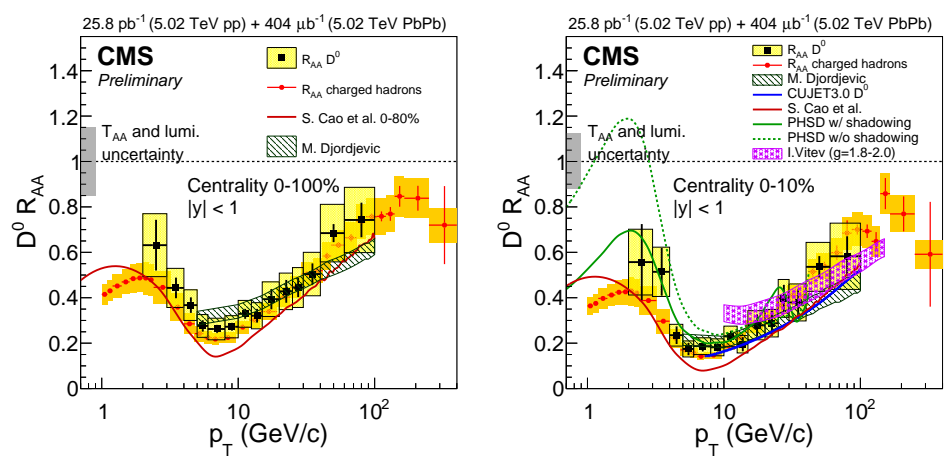

Figure 6: Prompt $D^{0} R_{A A}$ as a function of $p_{T}$ for centrality classes $0-100 \%$ (left) and $0-10 \%$ (right) in $\mathrm{PbPb}$ collision at 5.02 TeV. The charged particle $R_{A A}$ and model predictions are shown for comparison.

The left panel of Figure 7 shows nonprompt $\mathrm{J} / \psi R_{A A}$ as a function of $p_{T}$ in $\mathrm{PbPb}$ collisions at $2.76 \mathrm{TeV}$ [6]. The suppression of nonprompt $\mathrm{J} / \psi$ is found to be stronger with increasing $p_{T}$. The right panel shows prompt $D^{0} R_{A A}^{*}$, charged particle $R_{A A}$, and non-prompt $\mathrm{J} / \psi R_{A A}$ as function of $N_{\text {part }}$ in $\mathrm{PbPb}$ collisions at $2.76 \mathrm{TeV}$ [11], which indicates the prompt $D^{0}$ is more suppressed than non-prompt $J / \psi$. The results are consistent with calculations by Djordjevic et al. [12]. 

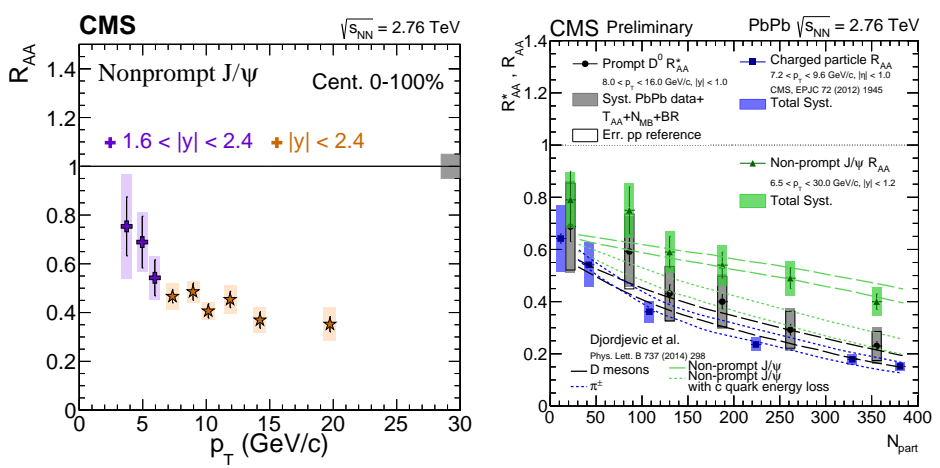

Figure 7: (Left) Nonprompt $\mathrm{J} / \psi R_{A A}$ as a function of $p_{T}$ in $\mathrm{PbPb}$ collisions at $2.76 \mathrm{TeV}$. (Right) Comparison of prompt $D^{0} R_{A A}^{*}$ with charged particle $R_{A A}$ and non-prompt $\mathrm{J} / \psi R_{A A}$ as function of $N_{\text {part }}$ in $\mathrm{PbPb}$ collisions at $2.76 \mathrm{TeV}$. The measurements are also compared with calculations.
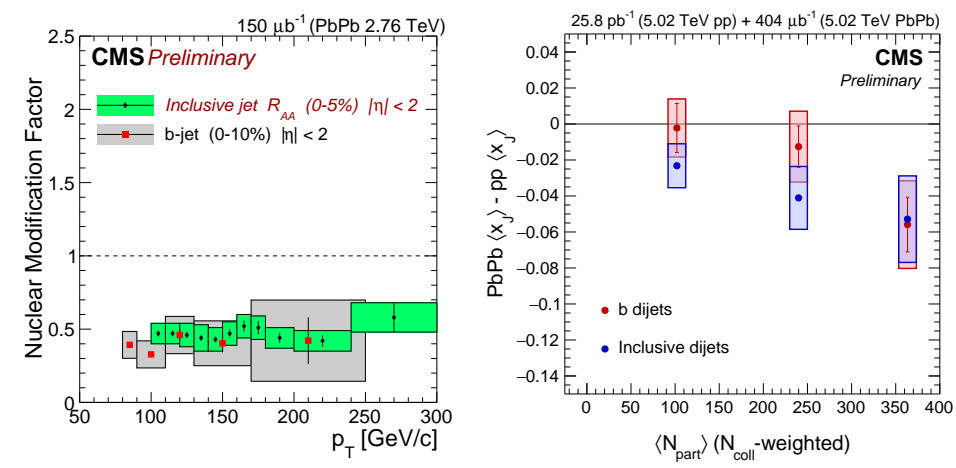

Figure 8: (Left) b-jet $R_{A A}$ as a function of $p_{T}$ for centrality class $0-10 \%$ in $\mathrm{PbPb}$ collisions at $2.76 \mathrm{TeV}$. The measurement of inclusive jet $R_{A A}$ is also plotted for comparison. (Right) Mean $x_{J}$ difference between $\mathrm{PbPb}$ and $\mathrm{pp}$ at $5.02 \mathrm{TeV}$ for inclusive dijets and $\mathrm{b}$ dijets for different centrality classes of $\mathrm{PbPb}$ collisions.

The left panel of Figure 8 shows the b-jet $R_{A A}$ as a function of $p_{T}$ for centrality class $0-10 \%$ in $\mathrm{PbPb}$ collisions at $2.76 \mathrm{TeV}$ [13]. It is found the $R_{A A}$ of b-jet is consistent with $R_{A A}$ of inclusive jets within uncertainties. Gluon splitting process is a big component and flavor creation process is not the dominant component in b-jet production at $2.76 \mathrm{TeV}$, thus it is difficult to conclude the flavor dependence energy loss from the comparsion of $R_{A A}$. To further study the flavor dependence energy loss, the b-dijets imbalance is studied in $\mathrm{PbPb}$ collisions at $5.02 \mathrm{TeV}$ and compared with the imbalance of inclusive dijets [14], where flavor creation process is the dominant component. The b-dijets imbalance is found to be at similar level as the inclusive dijets imbalance as shown in the right panel of Figure 8 .

\section{Summary}

This paper presents the CMS measurements of quarkonia and open heavy flavor in $\mathrm{pp}, \mathrm{pPb}$, and $\mathrm{PbPb}$ collisions at 2.76 and 5.02 TeV. Both cold nulcear matter effects and final state effects are 
studied with the measurements, which provides insight into the understanding of the interactions between heavy quarks and the medium.

\section{References}

[1] T. Matsui and H. Satz. J/ $\psi$ Suppression by Quark-Gluon Plasma Formation. Phys. Lett., B178:416-422, 1986.

[2] Yuri L. Dokshitzer and D. E. Kharzeev. Heavy quark colorimetry of QCD matter. Phys. Lett., B519:199-206, 2001.

[3] F. Dominguez, D. E. Kharzeev, E. M. Levin, A. H. Mueller, and K. Tuchin. Gluon saturation effects on the color singlet $\mathrm{J} / \psi$ production in high energy dA and AA collisions. Phys. Lett., B710:182-187, 2012.

[4] Serguei Chatrchyan et al. Event activity dependence of $\mathrm{Y}(\mathrm{nS})$ production in $\sqrt{s_{N N}}=5.02 \mathrm{TeV}$ $\mathrm{pPb}$ and $\sqrt{s}=2.76 \mathrm{TeV}$ pp collisions. JHEP, 04:103, 2014.

[5] $\mathrm{J} / \psi$ production in $\mathrm{pPb}$ collisions. Technical Report CMS-PAS-HIN-14-009, CERN, Geneva, 2015.

[6] Vardan Khachatryan et al. Suppression and azimuthal anisotropy of prompt and nonprompt $J / \psi$ production in $\mathrm{PbPb}$ collisions at $\sqrt{s_{N N}}=2.76 \mathrm{TeV} .2016$.

[7] Nuclear modification of $\mathrm{Y}$ states in PbPb. Technical Report CMS-PAS-HIN-15-001, CERN, Geneva, 2015.

[8] Vardan Khachatryan et al. Study of B Meson Production in $\mathrm{p}+\mathrm{Pb}$ Collisions at $\sqrt{s_{N N}}=5.02$ TeV Using Exclusive Hadronic Decays. Phys. Rev. Lett., 116(3):032301, 2016.

[9] Vardan Khachatryan et al. Transverse momentum spectra of inclusive $\mathrm{b}$ jets in $\mathrm{pPb}$ collisions at $\sqrt{s_{N N}}=5.02 \mathrm{TeV}$. Phys. Lett., B754:59, 2016.

[10] $\mathrm{D}^{0}$ meson nuclear modification factor in $\mathrm{PbPb}$ collisions at $\sqrt{s_{\mathrm{NN}}}=5.02 \mathrm{TeV}$. Technical Report CMS-PAS-HIN-16-001, CERN, Geneva, 2016.

[11] Nuclear Modification Factor of prompt $D^{0}$ in PbPb Collisions at $2.76 \mathrm{TeV}$. Technical Report CMS-PAS-HIN-15-005, CERN, Geneva, 2015.

[12] Magdalena Djordjevic, Marko Djordjevic, and Bojana Blagojevic. RHIC and LHC jet suppression in non-central collisions. Phys. Lett., B737:298-302, 2014.

[13] Serguei Chatrchyan et al. Evidence of b-Jet Quenching in $\mathrm{PbPb}$ Collisions at $\sqrt{s_{N N}}$ $=2.76$ TeV. Phys. Rev. Lett., 113(13):132301, 2014. [Erratum: Phys. Rev. Lett.115,no.2,029903(2015)].

[14] Transverse momentum balance of b-jet pairs in $\mathrm{PbPb}$ collisions at $5 \mathrm{TeV}$. Technical Report CMS-PAS-HIN-16-005, CERN, Geneva, 2016. 